REGARDS

SUR LECONOMIE ALLEMANDE

BULLETIN ECONOMIQUE DU CRAC

\section{Regards sur l'économie allemande}

Bulletin économique du CIRAC

$94 \mid 2009$

Varia

\title{
Vrais et faux enjeux de la controverse sur les salaires minima légaux en RFA
}

\section{Karl Brenke}

Traducteur : Isabelle Bourgeois

\section{OpenEdition \\ Journals}

Édition électronique

URL : http://journals.openedition.org/rea/3990

DOI : 10.4000/rea.3990

ISBN : 978-2-8218-0884-3

ISSN : 1965-0787

Éditeur

CIRAC

Édition imprimée

Date de publication : 1 décembre 2009

Pagination : 19-32

ISSN : 1156-8992

\section{Référence électronique}

Karl Brenke, «Vrais et faux enjeux de la controverse sur les salaires minima légaux en RFA », Regards sur l'économie allemande [En ligne], 94 | décembre 2009, mis en ligne le 01 décembre 2011, consulté le 19 avril 2019. URL : http://journals.openedition.org/rea/3990 ; DOI : 10.4000/rea.3990 


\section{Vrais et faux enjeux de la controverse sur les salaires minima légaux en RFA}

\author{
Karl Brenke
}

Karl Brenke, sociologue, est conseiller scientifique du président de l'Institut DIW (Berlin), où il est plus particulièrement en charge du marché de l'emploi, des revenus, de la conjoncture et de la politique régionale.

\section{Des salaires minima légaux existent jusqu'ici dans quelques rares branches seulement}

A la différence de la France, les salaires minima légaux ne sont pas la règle en République fédérale. Tout au long des décennies, les syndicats ouest-allemands avaient défendu avec acharnement l'autonomie tarifaire que leur garantit la Loi fondamentale, luttant contre toute tentative d'intervention de l'Etat dans la fixation des salaires. II n'en allait pas de même en RDA, où c'était de fait l'Etat qui fixait les salaires et où avait été adopté dès les années 1950 un seuil minimum légal. Au moment de la chute du Mur, celui-ci s'élevait à 400 marks est par mois pour les salariés à temps plein, soit environ $40 \%$ du salaire moyen des ouvriers et employés en 1989. Mais ce seuil minimum légal n'en restait pas moins largement théorique, très peu d'Allemands de l'Est le percevant.

\section{Le précédent du BTP}

Les premiers salaires minima légaux n'ont été introduits dans l'Allemagne unifiée qu'au milieu des années 1990 ; et encore, par des procédures administratives bien particulières. Si c'est dans le BTP qu'il s'est pour la première fois appliqué au niveau d'une branche, il avait été introduit peu auparavant dans un métier, très localisé et à faible effectif : celui des pilotes dans le port de Hambourg. L'objectif
Avant 1990, seule la RDA connaît un seuil minimum légal

Après l'Unité, adoption d'un salaire minimum légal d'abord au niveau catégoriel... 
... puis au niveau d'une branche : le BTP

Une finalité protectionniste

La tertiarisation met à mal le pouvoir syndical, ... de son introduction, dans ce cas précis, était de protéger les pilotes hambourgeois de la concurrence de leurs collègues est-allemands que le recul des activités portuaires dans l'ex-RDA incitait à chercher du travail à l'ouest, même à des rémunérations inférieures à celles habituellement pratiquées dans les ports ouest-allemands.

Dans la branche du BTP, un salaire minimum légal a été introduit en réponse à l'afflux croissant sur le marché allemand, principalement dans l'industrie du bâtiment, de salariés issus d'autres pays, essentiellement d'Europe de l'Est. C'est à cette époque qu'avait été adoptée la Loi sur les travailleurs détachés (Arbeitnehmer-Entsendegesetz, AEntG) fixant le cadre réglementaire des conditions de travail applicables aux étrangers occupant un emploi salarié en Allemagne. Or les dispositions de cette loi s'appliquaient de ce fait aussi aux salariés domiciliés en RFA. Mais elle ne comprenait pas de seuils salariaux minima. II fut d'abord tenté de les introduire via la convention tarifaire conclue en 1996 par les partenaires sociaux de la branche du BTP et qui, pour la première fois dans l'histoire des accords de branche en RFA, fixait des salaires minima. Cette convention devait ensuite être soumise au ministère fédéral du Travail pour bénéficier de la procédure d'extension (Allgemeinverbindlichkeitserklärung); de la sorte, elle aurait été contraignante pour l'ensemble de la branche du BTP, et pas seulement pour les seules entreprises affiliées à la fédération patronale du BTP. Or la Loi sur les conventions tarifaires (Tarifvertragsgesetz) prévoit que les décisions en la matière doivent être prises conjointement par le ministère, les syndicats et la ou les fédération(s) patronale(s) concernée(s). Les diverses fédérations patronales œuvrant dans la branche du BTP auraient certes donné leur accord à la procédure d'extension, mais la confédération du patronat BDA (Bundesvereinigung der deutschen Arbeitgeberverbände) y était opposée - or la réglementation lui donnait à l'époque voix prépondérante. Le politique modifia donc la Loi $A E n t G$ afin de permettre au ministère fédéral du Travail d'appliquer par décret la procédure d'extension à toute convention tarifaire - même en cas d'opposition de la part des fédérations patronales (ou des syndicats). Voilà comment on en vint à l'institution de salaires minima légaux dans la branche du BTP.

Les motivations qui y avaient poussé étaient clairement protectionnistes ; il s'agissait de barrer la route à la concurrence étrangère - principalement celle des entreprises et des salariés est-européens. Mais ces salaires minima s'appliquaient pareillement aux ressortissants des Etats membres que comptait l'UE à l'époque. Etaient tout particulièrement visés par exemple les carreleurs portugais auxquels recouraient de plus en plus les entreprises allemandes. Lors de la procédure d'extension, on fixa également en Allemagne de l'est des salaires minima inférieurs au niveau ouest-allemand afin de tenir compte des différences régionales en matière de salaires. Mais avec pour conséquence que les entreprises est-allemandes ne pouvaient intervenir sur les chantiers ouest-allemands que si les salaires qu'elles versaient à leurs salariés n'étaient pas inférieurs aux seuils en vigueur à l'ouest. De fait, par cette disposition, les entreprises du BTP d'Allemagne de l'ouest s'étaient mises à l'abri de leurs concurrentes est-allemandes.

\section{Recul du taux de syndicalisation et écartement de la fourchette salariale}

Le débat sur les salaires minima légaux ne resurgira qu'à partir de 2003/04. Cette fois, il a pour point de départ l'écartement de la fourchette des salaires : non seulement les disparités salariales s'étaient accrues au fil des ans, mais c'est surtout le segment des emplois faiblement rémunérés qui gagnait en importance. II y avait plusieurs raisons à cela. D'une part, la persistance d'un fort taux de chômage tassait globalement le niveau des salaires, essentiellement parce que, une large partie des chômeurs n'ayant pas de qualification professionnelle, ceux-ci ne pouvaient retrouver qu'un emploi faiblement rémunéré. S'ajoutent à cela les mutations sectorielles de l'activité qui, au fil des décennies, réduisaient la part du secteur manufacturier au profit du tertiaire. De ce fait, le pouvoir syndical, qui se fondait auparavant sur l'organisation des grandes entreprises industrielles, a vu 
se réduire sa base traditionnelle. Mais dans le même temps, les syndicats éprouvent de grandes difficultés à organiser un secteur des services en plein essor, essentiellement parce que les salariés y sont employés majoritairement dans de petites entreprises et que par ailleurs la culture d'entreprise comme celle des salariés y est très différente de celle qui marque le secteur productif.

Le pouvoir des syndicats s'est trouvé affaibli encore plus par l'évolution de la situation dans l'ex-RDA. Immédiatement après la chute du Mur, ils y avaient conclu des conventions tarifaires prévoyant une élévation des salaires est-allemands au niveau de ceux de l'ouest en quelques années seulement. Mais comme ces accords programmaient littéralement une hausse exorbitante des salaires que seules quelques rares entreprises étaient en mesure de supporter sans mettre en danger leur viabilité, il s'ensuivit une vague massive de désyndicalisation patronale ; quant à la plupart des entreprises nouvellement créées dans la phase de transition, elles refusaient d'adhérer aux fédérations patronales, ce qui leur permettait d'échapper au domaine d'application des conventions tarifaires. Cette vague de désyndicalisation patronale s'est ensuite étendue à l'Allemagne de l'ouest. Les salariés se sont largement accommodés de cette situation étant donné que le haut niveau de chômage ne les mettait pas en position de force pour négocier. Ils l'étaient d'autant moins que, aux côtés des traditionnels (et puissants) syndicats unitaires de branche naissait, à l'est d'abord, de nouveaux syndicats qui ont ensuite étendu de plus en plus leur rayon d'action à l'ouest.

S'y ajoute un autre élément : la montée en puissance des « mini-jobs », c'est-àdire de contrats de travail dispensant les salariés de verser impôts et cotisations sociales dès lors que la rémunération mensuelle est inférieure à $400 €$; quant aux charges de l'employeur, elles sont ramenées à un forfait réduit. Tout salarié peut cumuler plusieurs de ces mini-jobs; et leur rémunération reste exonérée d'impôts même si les autres membres du foyer tirent leurs revenus d'un emploi salarié. Ce type de contrat de travail s'est largement diffusé en Allemagne, ce qui n'a rien d'étonnant, puisqu'il bénéficie d'un régime particulier : il est subventionné par l'Etat. Pour le salarié, le salaire net est donc identique au brut, et pour l'employeur, ce régime présente de grands avantages en termes de coûts salariaux. Aux côtés de ces mini-jobs existent les « midi-jobs » où les salaires sont compris entre $400 €$ et $800 €$ par mois, et où les prélèvements s'accroissent avec le montant du salaire ; leurs taux restent néanmoins inférieurs à ceux prélevés sur les emplois réguliers soumis à cotisations sociales. Ces mini-jobs et midi-jobs, entièrement ou en partie exonérés de prélèvements, ont donc en toute logique contribué à leur tour à tasser le niveau des salaires versés dans les emplois réguliers - et ce, surtout dans le segment des emplois faiblement qualifiés.

\section{Extension des salaires minima à d'autres branches et cas particulier des activités postales (acheminement des lettres)}

Ces dernières années, la Loi sur les travailleurs détachés (AEntG) qui avait institué des salaires minima dans le BTP en a introduit dans d'autres secteurs. Mais ceux-ci comptant peu de salariés, cette tendance reste marginale. II s'agit essentiellement d'activités artisanales comme la peinture/plâtrerie, la couverture, l'électricité, l'entretien des bâtiments, une partie des teintureries et des services spécialisés dans le secteur minier (tableau 1). Les salaires minima fixés sont différents selon qu'il s'agit des Länder de l'est ou de l'ouest, et distinguent encore souvent entre salariés qualifiés ou non. Actuellement, ces salaires minima horaires - bruts - s'établissent dans une fourchette allant de 6,36 € (teinturerie, Allemagne de l'est) à 12,90 € (BTP, ouvriers professionnels, ouest).

Le salaire minimum légal adopté pour les activités d'acheminement des lettres constitue un cas particulier. Au $1^{\mathrm{er}}$ janvier 2008, la Deutsche Post AG, opérateur historique autrefois public, a perdu presque en totalité son monopole dans le segment de la lettre. Certes, dès avant cette date, les concurrents privés pouvaient distribuer des lettres, mais à condition de fournir des services dits « de qualité supérieure » (par exemple collecte chez le client ou la livraison express le même
... de même qu'un rattrapage salarial disproportionné à l'est...

... puis la montée en puissance des mini-jobs

L'extension des salaires minima reste marginale

Le secteur postal reste un cas particulier.. 
... qui occupe actuellement la jurisprudence

jour), ce qui était le plus souvent économiquement hors de portée des nouveaux entrants et limitait de ce fait l'émergence de la concurrence. Ces conditions particulières ont été levées au début 2008. Mais l'opérateur historique bénéficie toujours de l'exonération de la TVA, qui sera néanmoins restreinte à la distribution du courrier chez les particuliers à dater du $1^{\mathrm{er}}$ janvier 2010. Autrement dit, Deutsche Post AG jouit toujours d'un avantage compétitif sur ses concurrents.

La perspective de la levée du monopole postal avait donc incité quelques grands groupes, notamment des groupes de presse, à investir le secteur de la lettre. A la suite de quoi l'opérateur en situation de monopole avait conclu avec le syndicat qui était autrefois l'unique représentant des salariés du secteur postal une convention tarifaire fixant des salaires minima. Toutefois, de leur côté, les opérateurs concurrents avaient conclu avec un syndicat nouvellement créé une convention tarifaire établissant elle aussi des salaires minima légaux, mais à un niveau inférieur à ceux préconisés par Deutsche Post AG [voir sur ce sujet $R E A$ 85/08; IB]. Après quelques tractations, le gouvernement de Grande coalition sortant a finalement appliqué la procédure d'extension à la convention conclue par l'opérateur anciennement en situation de monopole. La conséquence en a été que les concurrents ont fermé la plupart de leurs sites et détruit une part importante de leurs emplois. L'affaire n'est pas close, le Tribunal administratif fédéral (BVerwG) devant encore statuer sur la validité de la procédure d'extension après que, en première instance, le tribunal administratif de Berlin avait considéré celle-ci comme invalide. Mais tant que l'affaire n'aura pas été tranchée en dernière instance, la convention tarifaire de Deutsche Post AG a force de loi pour l'ensemble du secteur. A l'expectative juridique s'ajoute l'incertitude quant à la décision que prendra le nouveau gouvernement de coalition Union/FDP à l'issue de l'expiration de la convention tarifaire de l'opérateur historique.

Tableau 1 : Salaires minima bruts horaires en RFA (novembre 2009)

\begin{tabular}{|c|c|c|c|}
\hline & Länder de l'ouest & Berlin & Länder de l'est \\
\hline \multicolumn{4}{|l|}{ BTP } \\
\hline Ouvriers non qualilfiés, conducteurs d'engin & 10,80 & 10,80 & 9,25 \\
\hline Ouvriers spécialisés, machinistes, chauffeurs & 12,90 & 12,75 & - \\
\hline \multicolumn{4}{|l|}{ Peinture/plâtrerie } \\
\hline Salariés non qualifiés & 9,50 & 9,50 & 9,50 \\
\hline Ouvriers qualifiés, compagnons (artisans qualifiés) & 11,25 & 11,25 & 9,50 \\
\hline Couverture & 10,40 & 10,40 & 10,40 \\
\hline Electricité (artisanat) & 9,55 & 8,05 & - \\
\hline \multicolumn{4}{|l|}{ Entretien des bâtiments ${ }^{\text {a) }}$} \\
\hline Hygiène et nettoyage des locaux (surfaces) & 8,15 & 8,15 & 6,58 \\
\hline Nettoyage des bâtiments (vitres et façades) & 10,80 & 8,34 & 8,01 à 8,34 b) \\
\hline \multicolumn{4}{|l|}{ Services postaux } \\
\hline Distribution du courrier & 9,80 & 9,80 & 9,00 \\
\hline Autres tâches & 8,40 & 8,40 & 8,00 \\
\hline Teinturerie & 7,51 & 6,36 & 6,36 \\
\hline \multicolumn{4}{|l|}{ Activités spécialisées dans le secteur minier (houille) } \\
\hline Haveurs/raboteurs (compétences techniques spécialisées) & 12,41 & 12,41 & 12,41 \\
\hline Abatteurs/piqueurs (ouvriers non qualifiés) & 11,17 & 11,17 & 11,17 \\
\hline
\end{tabular}

Source : Destatis. a) la convention tarifaire a expiré le 30-09-2009 ; b) variable selon les Länder.

Finalité : limiter la concurrence dans un secteur en voie de libéralisation
Or dans le domaine postal comme naguère dans le BTP, la fonction première de la convention introduisant un salaire minimum est de limiter la concurrence. Certes, on pourrait avancer l'argument que les accords sur les salaires minima créent un cadre concurrentiel identique pour tous les acteurs du marché. Or cette situation n'est pas donnée lorsqu'un opérateur historique domine un marché en voie de libéralisation. En effet, les nouveaux entrants peinent à s'établir sur le marché de la distribution de la lettre face à l'opérateur anciennement en situation de monopole étant donné que les coûts sont plus élevés pour eux dans la phase de lancement des activités. En particulier, ils ne disposent pas d'un réseau de distribution de la même densité et donc d'une clientèle limitée en comparaison de 
Deutsche Post. Pour perdurer, ils doivent donc miser sur les avantages coûts qu'ils peuvent avoir dans d'autres domaines - et dans les secteurs intensifs en main-d'œuvre, ce sont alors principalement les salaires, puisqu'ils n'ont guère de marge de manœuvre en ce qui concerne les prix qu'ils peuvent facturer aux clients pour la distribution des lettres; ceux-ci sont limités par le prix du timbre de Deutsche Post AG. En un mot : pour les nouveaux entrants, les salaires minima constituent une entrave à la concurrence - et pas seulement sur des marchés à la structure anciennement monopolistique ou oligopolistique.

\section{Etat des lieux du débat actuel sur les salaires minima légaux}

\section{Ceux qui plaident pour et ceux qui s'y opposent}

Bien que l'instauration de salaires minima soit revendiquée avec une véhémence croissante, ceux-ci ne jouent qu'un rôle marginal dans la pratique des rémunérations en Allemagne. Et il n'est actuellement pas prévu d'adopter des 'SMIC' uniformes, légaux, tels qu'ils existent dans de nombreux Etats membres de l'UE - et tels que les préconisent un certain nombre d'acteurs : tout particulièrement le parti Die LINKE qui trouve son origine dans le parti communiste de la RDA, ainsi qu'une partie du mouvement syndical, principalement dans le secteur des services. S'y ajoute depuis peu le parti social-démocrate bien que, au cours des onze années où il fut au gouvernement, il ne se soit pas très activement impliqué en la matière. Le parti Die Grünen plaide lui aussi aujourd'hui pour un salaire minimum légal généralisé bien que, tout comme le SPD avec lequel il partageait le pouvoir jusqu'en 2005, il ne se soit alors guère intéressé à la question. De leur côté, les syndicats d'industrie se montrent relativement indifférents à la thématique, ce qui s'explique par le niveau comparativement élevé des salaires pratiqués dans leurs branches. Enfin, s'opposent à tout seuil salarial plancher les partis conservateurs (CDU, CSU) et libéral (FDP).

Les opposants estiment que la généralisation de tels seuils détruit l'emploi. Ceux qui plaident pour avancent une série d'arguments : le salaire minimum devrait atteindre un montant suffisant pour permettre au salarié d'assurer sa subsistance ; son instauration aiderait à réduire l'inégalité croissante des revenus; enfin, elle augmenterait le pouvoir d'achat dans la tranche des bas salaires, ce qui accrôitrait la consommation des ménages et aurait un effet favorable sur la croissance. Ces arguments reposent implicitement sur l'idée que l'Etat doit intervenir dans le processus de fixation des salaires parce que les syndicats sont aujourd'hui trop affaiblis pour exercer cette responsabilité. Considérons de plus près les arguments avancés pour ou contre un 'SMIC' légal en Allemagne.

\section{Les salaires minima sont-ils justifiés sous l'angle de la politique sociale ?}

Selon la définition communément utilisée, celle de l'OCDE, constituent le secteur des bas salaires toutes les activités où les rémunérations sont inférieures aux deux tiers du salaire médian. Rappelons ici la distinction entre salaire moyen et salaire médian : ce dernier constitue le niveau séparant les salariés en deux moitiés, l'une percevant des salaires supérieurs à ce médian, l'autre, des salaires inférieurs. En Allemagne, selon les données du Panel Socio-économique SOEP (il s'agit d'une enquête annuelle menée dans la population par l'institut DIW en coopération avec TNS Infratest), le salaire médian horaire s'établissait en 2008 à $13,41 €$ bruts. Le secteur des bas salaires s'étend donc à toutes les activités rémunérées à moins de $8,95 €$ par heure. Pour en revenir aux débats sur le salaire minimum légal : les seuils revendiqués se situent généralement soit à $7,50 €$ (syndicats et, désormais, SPD) soit à $10 €$ (Die LINKE).

En Allemagne, un bon cinquième des actifs est occupé dans le secteur à bas salaires, c'est-à-dire de moins de $8,95 €$ bruts par heure (tableau 2). Si étaient instaurés des salaires minima horaires de $7,50 €$, alors ceux-ci bénéficieraient à un actif sur sept; si ce montant était de $10 €$, il concernerait plus d'un actif sur
Pour : la gauche et le tertiaire ; contre : les partis de l'actuelle coalition

Les arguments avancés par les uns et les autres

Médian horaire brut : $13,41 €$ Bas salaires : inférieurs à $8,95 €$

Un actif sur 5 est occupé dans le segment des bas salaires 
Essentiellement des emplois atypiques

Peu de bas salaires dans l'emploi régulier

quatre. II convient néanmoins de distinguer entre les différentes régions et les divers groupes de salariés. Ainsi, par exemple, les salaires minima auraient un impact supérieur dans les nouveaux Länder que dans les anciens : à l'est, un seuil à $7,50 €$ entraînerait une hausse des salaires bruts pour un quart des salariés, un seuil de $10 €$ pour la moitié d'entre eux, alors qu'à l'ouest ne seraient concernés respectivement que moitié moins de salariés. Cela reflète les fortes disparités salariales qui perdurent jusqu'à aujourd'hui entre l'est et l'ouest : selon les données établies par Destatis, le salaire horaire brut moyen n'atteignait dans les nouveaux Länder que $74 \%$ du niveau ouest-allemand.

Mais il n'y a pas que ces grandes différences entre l'est et l'ouest. Car la structure même de l'emploi révèle que ce sont surtout les actifs occupant un emploi atypique qui perçoivent les bas salaires et que ce sont donc eux que concernerait en premier lieu l'adoption de salaires minima généralisés. Ces actifs sont des lycéens ou des étudiants exerçant un petit boulot pour financer leurs études, des retraités qui arrondissent leurs fins de mois ou des chômeurs en quête de revenus complémentaires. Une partie des activités qu'exercent ces catégories relèvent du travail au noir. Enfin, l'emploi atypique inclut également les mini-jobs et les midijobs. Les salariés qui les occupent, c'est-à-dire qui ne sont ni retraités, étudiants ou chômeurs, sont eux aussi faiblement rémunérés.

II en va autrement des actifs occupant un emploi régulier. Dans ce groupe, les salariés en temps partiel et, plus encore, ceux en temps plein, ne sont que très peu nombreux en comparaison à entrer dans la catégorie des bas salaires. Certes, ici aussi, les différences est-ouest sont grandes. II n'en reste pas moins que, si le seuil des salaires minima était fixé à $7,50 €$, il faudrait augmenter les salaires bruts pour $5 \%$ des actifs en temps plein à l'ouest, et pour $20 \%$ à l'est. Un seuil de $10 €$ entraînerait même une hausse pour $15 \%$ (ouest) et 40 \% (est) des salariés.

Tableau 2 : Salariés * percevant des bas salaires (bruts, horaires) en 2008

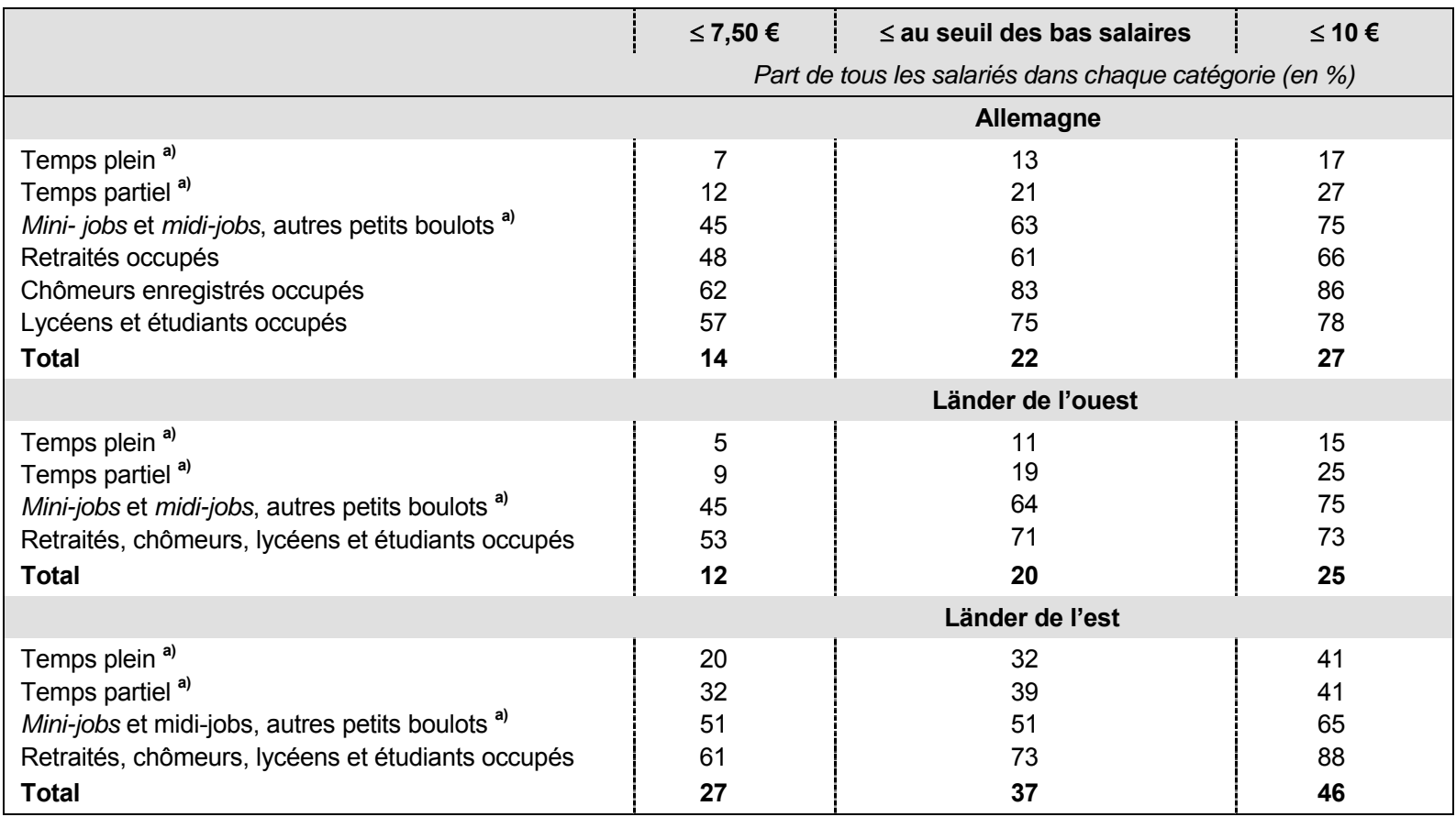

Source : SOEP ; calculs de l'auteur. *) hors apprentis, chômeurs occupant un « job à $1 €$ », jeunes appelés ou jeunes effectuant leur service civil ; a) hors retraités, chômeurs enregistrés, lycéens et étudiants occupés.

L'approche du socialement souhaitable...
Or les arguments de ceux qui plaident pour l'instauration de salaires minima légaux ne portent pas seulement sur le montant des salaires en tant que tel ; ils revendiquent aussi que ce montant soit suffisant pour permettre au salarié d'assurer seul sa subsistance, c'est-à-dire sans être tributaire d'une aide fournie par l'Etat. Cette revendication est à l'évidence motivée par des considérations 
de politique sociale, car elle repose sur l'idée que le montant du salaire doit prendre pour référence les besoins du salarié et non pas s'établir sur la base de critères économiques comme l'offre et la demande sur le marché de l'emploi ou, ce qui en est le corollaire, la productivité des salariés.

Or cette approche ne tient compte en rien du fait que ces besoins peuvent être extrêmement variables, et que donc, en toute logique, les mêmes emplois devraient donner lieu à des rémunérations d'un niveau lui aussi variable. En effet, le chef d'une famille nombreuse où la mère n'occupe pas d'emploi a indéniablement des besoins supérieurs à ceux d'un célibataire. Celui qui a ainsi plusieurs personnes à sa charge au sein de son foyer devrait en conséquence percevoir un salaire plus élevé qu'un célibataire qui n'a à assurer que sa propre subsistance. Aussi absurde que puisse être l'idée d'un salaire qui serait fonction des besoins de celui qui le touche, elle n'en a pas moins une longue histoire : Karl Marx avait tourné en dérision une telle approche en 1875, à l'occasion du débat sur le programme de Gotha de la social-démocratie allemande. Car si l'aune à laquelle se mesurent les rémunérations était le besoin du salarié, alors les célibataires se verraient privilégiés à l'embauche - et à l'inverse, ceux qui ont plusieurs personnes à leur charge se verraient discriminés sur le marché de l'emploi.
... ne résiste pas face à des besoins extrêmement variables

\section{Marx, déjà, considérait cette approche égalitaire comme indéfendable}

« Le droit par sa nature ne peut consister que dans l'emploi d'une même unité de mesure ; mais les individus inégaux (et ce ne seraient pas des individus distincts, s'ils n'étaient pas inégaux) ne sont mesurables d'après une unité commune qu'autant qu'on les considère d'un même point de vue, qu'on ne les saisit que sous un aspect déterminé ; par exemple, dans le cas présent, qu'on ne les considère que comme travailleurs et rien de plus, et que l'on fait abstraction de tout le reste. D'autre part : un ouvrier est marié, l'autre non ; l'un a plus d'enfants que l'autre, etc., etc. A égalité de travail et par conséquent, à égalité de participation au fonds social de consommation, l'un reçoit donc effectivement plus que l'autre, l'un est plus riche que l'autre, etc. Pour éviter tous ces inconvénients, le droit devrait être non pas égal, mais inégal. Mais ces défauts sont inévitables... »

Karl Marx, Critique du programme de Gotha, in : Karl Marx, Friedrich Engels, Werke. Band 19, 4, Berlin 1962, pp.13-32. [NdT : texte français consultable sur : www.marxists.org/français/marx/works/1875/05/18750500.htm].

Or dans la réalité allemande, il n'existe de fait que très peu de salariés touchant un bas salaire et vivant dans un foyer où ils seraient les seuls à tirer leurs revenus d'un emploi salarié. Cela est particulièrement vrai si on considère les salariés à temps plein - les seuls auxquels pourrait s'appliquer (si tant est qu'elle le puisse) cette revendication mue par une approche de politique sociale : à savoir que les salaires doivent permettre de gagner assez pour en vivre. En revanche, les salariés qui se contentent d'un temps partiel ou qui exercent un mini-job n'ont aucune raison d'espérer percevoir, en contrepartie d'une durée de travail réduite, un salaire considéré ainsi comme suffisant à leurs besoins. Actuellement, en Allemagne, seuls $3 \%$ des actifs occupant un emploi à temps plein gagnent moins de 7,50 € et vivent dans un foyer dont ils assurent seul le revenu (tableau 3). Et même si on prend pour salaire plancher le seuil de $10 €$, ils ne sont au total que $8 \%$.
Les bas salaires sont le plus souvent des revenus complémentaires

Tableau 3 : Salariés ${ }^{*}$ percevant des bas salaires (bruts, horaires) en 2008 - et qui sont les seuls, dans leur foyer, à tirer leurs revenus d'un emploi salarié

\begin{tabular}{|l|c|c|c|}
\hline & $\leq \mathbf{7 , 5 0} €$ & $\leq$ au seuil des bas salaires & $\leq \mathbf{1 0} €$ \\
& Part de tous les salariés dans chaque catégorie (en \%) \\
Temps plein $^{\text {a) }}$ & 3 & 5 & 8 \\
Temps partiel $^{\text {a) }}$ & 3 & 5 & 6 \\
Mini- jobs et midi-jobs, autres petits boulots $^{\text {a) }}$ & 11 & 18 & 22 \\
Retraités, chômeurs enregistrés, lycéens et étudiants occupés & 30 & 41 & 43 \\
Total & $\mathbf{5}$ & $\mathbf{8}$ & $\mathbf{1 1}$ \\
\hline
\end{tabular}

Source : SOEP ; calculs de l'auteur. *) hors apprentis, chômeurs occupant un « job à $1 €$ », jeunes appelés ou jeunes effectuant leur service civil ; a) hors retraités, chômeurs enregistrés, lycéens et étudiants occupés.

Dans le débat sur les salaires minima, on évoque par ailleurs souvent ce groupe des «Aufstocker » (qui touchent des prestations en complément de leurs revenus), c'est-à-dire de ceux qui, tout en étant salariés, perçoivent des transferts so-

Les 1,3 million d'Allemands dont le salaire est complété par des prestations sociales ... 
... occupent le plus souvent un emploi à temps partie
Particularités allemandes

Les données internationales ne sont guère comparables entre elles

De fortes inégalités salariales...

... mais un salaire médian supérieur à la moyenne internationale ciaux complémentaires sous la forme du forfait Arbeitslosengeld /l créé par la Loi Hartz IV en 2005 parce que leurs revenus salariés seuls ne leur permettent pas d'assurer leur subsistance. En Allemagne, selon l'Agence fédérale pour l'Emploi, un peu plus de 1,3 million de personnes étaient bénéficiaires en juin 2009 de cette mesure comparable au revenu de solidarité active (RSA) français.

Dans l'écrasante majorité des cas, il s'agit toutefois de salariés qui, sous une forme ou une autre, occupent un emploi à temps partiel. II n'existe certes pas de statistiques officielles sur le temps de travail de ces "Aufstocker "; mais on connaît la structure de leurs revenus : ainsi, 760000 d'entre eux perçoivent un salaire mensuel de $400 €$ (le plafond des mini-jobs) et 240000 un salaire compris entre $400 €$ et $800 €$ (le périmètre des midi-jobs). Ce groupe de personnes travaille donc pour s'assurer un revenu venant en complément des prestations sociales qu'elles perçoivent. Quant au nombre de salariés qui, tout en occupant un emploi à temps plein, bénéficient de transferts sociaux pour assurer leurs ressources, il devrait s'établir au maximum à 300000 . Ce nombre n'est donc pas très important. Et il convient par ailleurs de rappeler qu'il y a toujours eu, en Allemagne, des personnes dont le revenu est insuffisant pour assurer leur subsistance et qui de ce fait bénéficient de prestations sociales (aide sociale complémentaire ou allocation logement, par exemple). En conséquence, on aurait dû voir s'élever dès les années 1970 ou 1980 des voix revendiquant l'introduction de salaires minima.

En un mot : l'argument selon lequel il faut instaurer des salaires minima pour éviter à certains actifs occupés de recourir aux prestations sociales repose sur une base empirique peu solide. On compte certes en Allemagne un nombre non négligeable de salariés percevant un faible salaire, mais ils se recrutent pour l'essentiel parmi ceux qui travaillent à temps réduit, de même que parmi les retraités, les étudiants et les chômeurs inscrits qui ont pris un emploi pour compléter leurs allocations. Mais il n'y a dans le segment des bas salaires que peu de salariés à temps plein vivant dans un foyer où personne d'autre qu'eux n'occupe un emploi salarié.

\section{L'Etat doit-il fixer des salaires minima pour éviter que s'accroissent les disparités salariales ?}

En comparaison internationale, l'évolution des salaires en Allemagne présente de nettes particularités. D'une part, la progression des salaires a été plus faible qu'ailleurs ; les salaires nets réels ont même baissé jusqu'en 2008 - et ce, plus fortement que dans les quelques Etats de l'UE où les salariés ont eux aussi subi des pertes de salaire net (Brenke, 2009). D'autre part, la fourchette salariale s'est écartée dans une proportion inconnue ailleurs.

Selon les données comparées fournies par Eurostat, l'écart salarial est particulièrement élevé en Allemagne. Précisons toutefois que les seules données disponibles concernent les salaires mensuels de 2006, et seulement ceux versés dans les entreprises de plus de dix salariés. Et comme le temps de travail hebdomadaire, et partant mensuel, varie selon les pays, ces variations mènent à quelques effets de distorsion lorsqu'on cherche à comparer les données entre elles. Si cela vaut pour le temps plein, cela est se révèle encore plus problématique pour comparer le temps partiel, étant donné que la durée du temps de travail dépend des préférences individuelles. Nous nous contenterons donc ici des données relatives aux salariés occupant un emploi à temps plein.

En ce qui concerne ces derniers, on constate qu'en 2006 les $10 \%$ des salariés allemands qui perçoivent les salaires les plus bas (jusqu'à $1247 €$ par mois) n'atteignent même pas la moitié du salaire médian (tableau 4). En France, les mêmes $10 \%$ inférieurs (dont les salaires mensuels vont jusqu'à 1429 $€$ ) touchent plus des deux tiers du salaire médian. Dans aucun des autres Etats de I'UE, les inégalités salariales sont aussi fortes qu'en Allemagne.

II faut cependant garder à l'esprit, dans cet exercice de comparaison, que le niveau des salaires dans la tranche des $10 \%$ inférieurs n'est pas particulièrement bas. La différence avec la France, par exemple, est certes de $182 €$, soit $13 \%$ de moins 
pour les Allemands ; mais comme le coût de la vie est inférieur en Allemagne, elle se réduit à $89 €(7 \%)$ si on la rapporte au pouvoir d'achat. Cet écart s'explique en partie par une durée hebdomadaire du travail plus longue en Allemagne; selon Eurostat, chez les salariés à temps plein, elle était habituellement de 39,1 heures en France en 2006, contre 40,3 heures en Allemagne (3\% de plus). Plus important que le niveau des bas salaires pour comprendre le fort écart salarial outre-Rhin est le fait que le salaire médian allemand est largement supérieur à la moyenne internationale. II se situe ainsi à plus de $500 €$ au-dessus du niveau français ; mais si on tient compte du pouvoir d'achat, il est de plus de $600 €$.

Tableau 4 : Les salaires perçus par les actifs occupés à temps plein dans l'UE (2006)

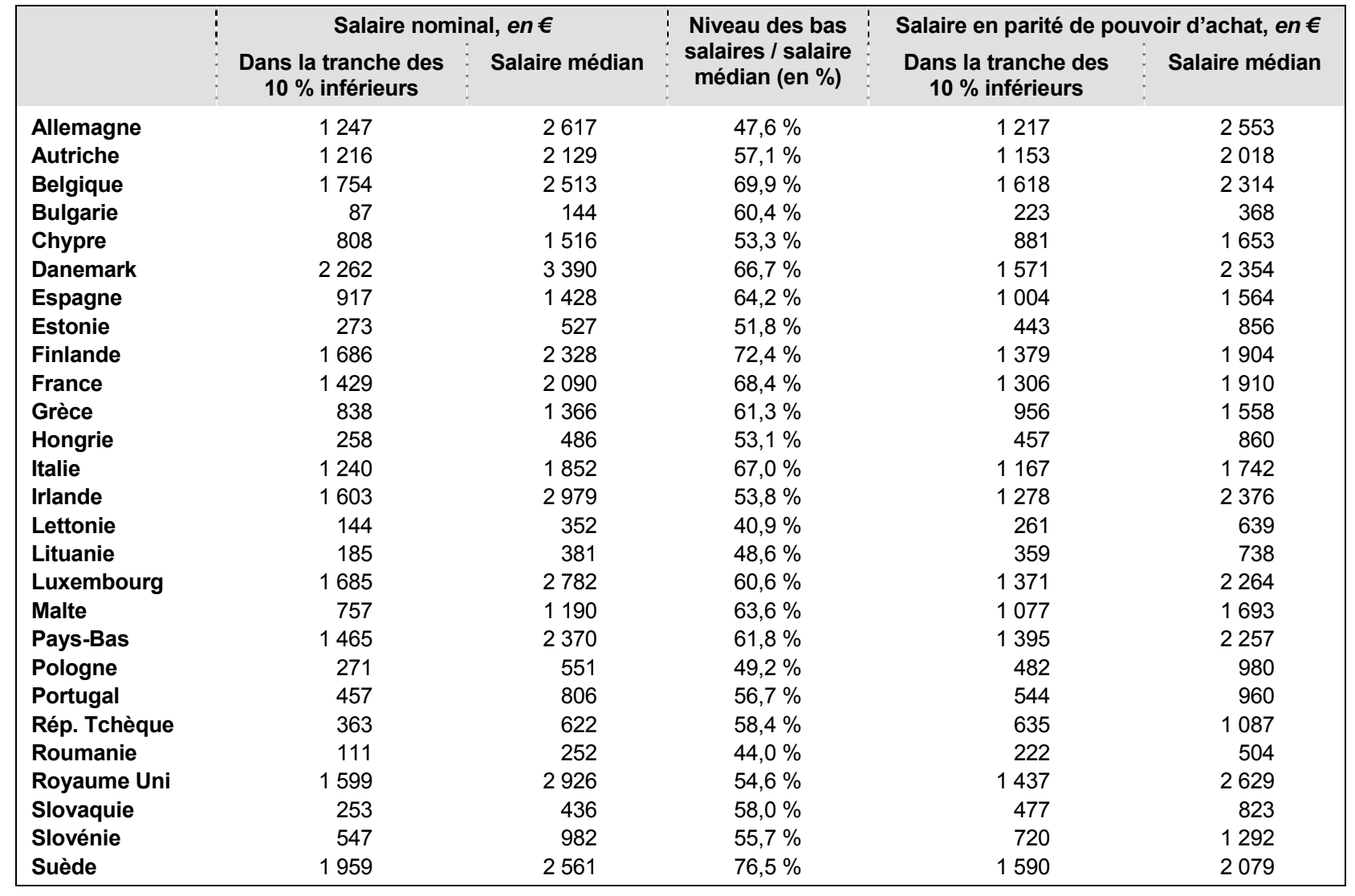

Source : Eurostat ; calculs de l'auteur.

Les données 2008 du SOEP relatives aux salaires horaires révèlent que le fort écart de la fourchette salariale en Allemagne résulte largement des différences dans les salaires versés à l'est et à l'ouest. Ainsi, dans les nouveaux Länder, le salaire d'un salarié à temps plein figurant parmi les $10 \%$ d'actifs aux revenus les plus bas n'atteint que $44 \%$ du salaire médian de tous les salaires (temps plein) en RFA ; à l'ouest, le salaire du même groupe en atteint un peu plus de $60 \%$. C'est certes moins qu'en France, mais cela correspond à la moyenne européenne - si on prend pour référence les salaires mensuels, les seuls pour lesquels il existe des données permettant des comparaisons internationales.

L'écart salarial se révèle encore plus net en Allemagne si on ne considère pas seulement les salariés à temps plein, mais aussi à temps partiel - surtout si on y intègre ceux qui occupent un mini-job, ainsi que les retraités, étudiants et chômeurs qui travaillent. Dans la catégorie des salariés à temps partiel et tout particulièrement des salariés occupant un emploi atypique, l'écart entre ceux qui appartiennent au groupe des plus bas salaires et le salaire médian est nettement plus prononcé que dans la catégorie des salariés à temps plein; et dans cette dernière aussi, on constate de fortes disparités entre l'est et l'ouest de l'Allemagne (graphique 1).
De fortes disparités régionales, ...

... surtout parmi les salariés à temps partiel 
Les disparités salariales relèvent de la normalité

Elles reflètent capacités et performances de l'économie

Sur les USA et la France,

Très peu de travaux sur leur impact en RFA

Graphique 1: Salaires des $10 \%$ des salariés *) aux revenus les plus bas par rapport au salaire médian (2008), en \%

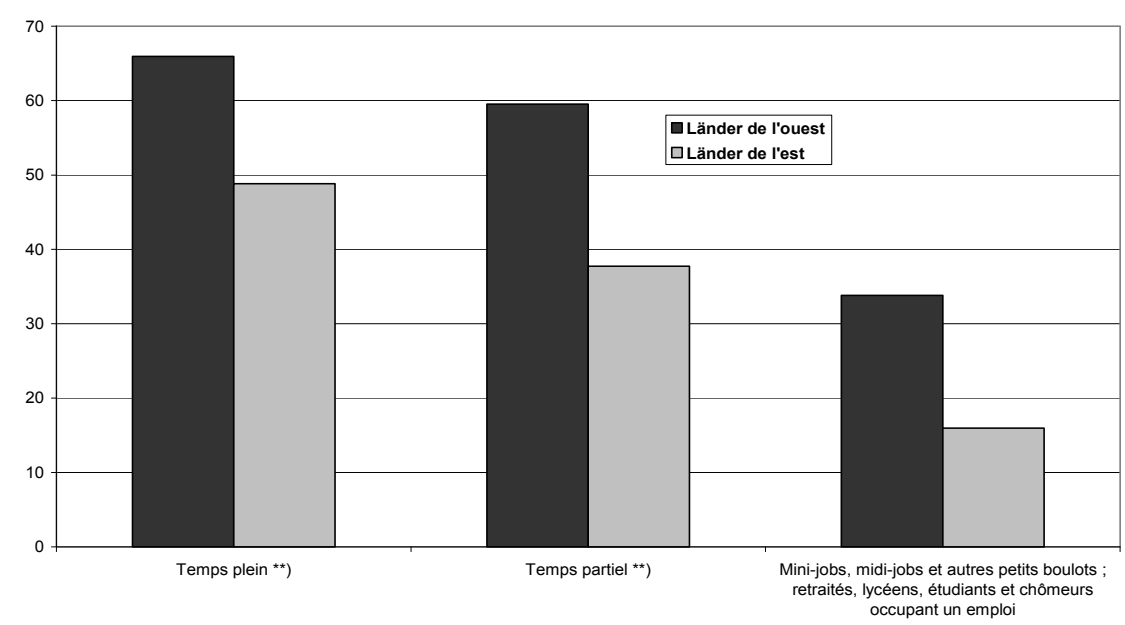

Source: SOEP, calculs de l'auteur. *) hors apprentis, chômeurs occupant un "job à $1 €$ », jeunes appelés ou jeunes effectuant leur service civil ; ${ }^{* *}$ ) hors retraités, chômeurs enregistrés, lycéens et étudiants occupés.

Considéré du point de vue économique, un écart salarial plus ou moins important ne peut en rien constituer un argument pour l'introduction de salaires minima, puisque les salaires sont le fruit d'une transaction effectuée par les salariés et les employeurs sur le marché du travail. Bien sûr, ce processus est déterminé par la puissance relative des partenaires de la négociation, et celle-ci dépend du contexte qu'offre l'évolution de la société. Ainsi, un fort taux de chômage affaiblit la position des syndicats ; à l'inverse, une phase de croissance de l'emploi renforce leur pouvoir dans la négociation. Pour ce qui est de l'Allemagne, il s'est ainsi avéré que la dernière phase de croissance et le net recul du chômage qui l'accompagnait ont mis fin à la tendance à l'écartement de la fourchette salariale (Brenke, 2008). Le meilleur moyen pour lutter contre une hausse des disparités salariales réside donc dans l'accroissement de l'offre d'emplois et donc celle de percevoir des revenus salariés.

Dans un débat sur des salaires minima, il convient de toujours garder présent à l'esprit que le niveau des salaires doit être le reflet des capacités et performances de l'économie. Cela vaut tout particulièrement pour l'Allemagne où, après la chute du Mur, il a fallu, sur le territoire de l'ex-RDA, reconstruire presque entièrement les bases mêmes de l'économie. Mais le processus de transition est loin d'être achevé : en 2008, la productivité horaire n'y atteint toujours que près des trois quarts du niveau ouest-allemand - ce qui se traduit pareillement dans l'écart des salaires est-ouest.

\section{L'instauration de salaires minima généralisés est-elle destructrice d'emplois ?} ils sont peu probants

Les opposants à l'instauration de salaires minima généralisés redoutent que ceuxci détruisent des emplois. La littérature scientifique consacrée aux effets de tels salaires sur l'économie allemande est peu abondante à ce jour, ce qui n'a rien d'étonnant, puisque les salaires minima sont l'exception. Généralement, on s'est contenté d'étudier sous l'angle macro-économique leurs effets sur l'emploi - pour le cas où serait instauré en Allemagne un salaire minimum légal (voir notamment Müller et al., 2008 ; .Bachmann et al., 2008 ; Ragnitz et al., 2008). Certains de ces travaux se penchent également sur des questions comme leurs effets sur l'encours fiscal. Quelques rares études se consacrent à leur impact sur certains segments de l'économie, sur les PME (Meyer et al., 2007) ou le BTP (König/Möller, 2007)

Aussi peu convaincant que sur l'Allemagne est l'état de la recherche sur d'autres pays. A cet égard, les Etats-Unis, où il existe une très abondante littérature sur l'effet des salaires minima, fournissent un exemple patent. Car si dans leur majorité, les travaux parviennent à la conclusion que les salaires minima compriment 
l'emploi, d'autres leur accordent des effets neutres, voire positifs (pour un état des lieux de ces publications, voir Neumark/Wascher, 2007). Mais le plus souvent, cette littérature n'apporte pas de conclusions probantes. II en va de même de la recherche en France, où les travaux sont loin d'être univoques (pour un état des lieux, voir Sturm, 2008). Tout au plus a-t-on pu prouver l'effet néfaste des SMIC sur l'emploi des jeunes et des jeunes adultes.

Ces divergences s'expliquent surtout par le recours à des méthodologies différentes. En effet, le problème vient de ce qu'il n'existe pas, jusqu'à présent, une méthode unanimement reconnue pour évaluer l'impact des salaires minima. II n'y a rien d'étonnant à cela, tant sont nombreux les facteurs à prendre en considération ; or non seulement on les connaît mal, mais encore on ignore presque tout de la manière dont ils peuvent interagir. Ainsi par exemple sont peu étudiées les réactions des salariés et, plus encore, des employeurs, à l'instauration de salaires minima ; ou encore, sous l'angle macro-économique, les répercussions de ces derniers sur l'évolution des bénéfices et des prix, du comportement des consommateurs et, plus généralement, de la demande (en particulier de celle des salariés rémunérés au 'SMIC'), ainsi que sur la structure des salaires. La plupart du temps, les travaux sur les salaires minima ne prennent guère en considération ces aspects, se contentant d'en analyser l'impact sous un angle réducteur : ils se concentrent sur un point particulier isolé d'une réalité pourtant complexe. Et trop souvent, ils se basent sur des hypothèses rarement vérifiées empiriquement pour se livrer à l'évaluation de certains des effets évoqués ci-dessus. S'ajoute à cela que les données prises en considération sont elles-mêmes hautement problématiques : ni la recherche empirique ni l'approche en matière de théorie économique ne sont univoques. Dès lors, les conclusions quant aux effets sur l'emploi que peuvent avoir les salaires minima sont fonction de la modélisation utilisée ; or ces modélisations livrent une lecture abstraite de la réalité et sont donc presque toujours impossibles à vérifier empiriquement.

Pour illustrer cette problématique, prenons par exemple l'argumentation suivante utilisée par ceux qui plaident pour l'instauration de salaires minima et selon laquelle celle-ci aurait un effet bénéfique sur la consommation, et donc la demande intérieure.

II est certain que ceux qui verront de la sorte augmenter leur salaire auront un pouvoir d'achat accru ; et tout aussi assurément, ils devraient consommer plus, sachant que dans le segment des bas salaires, le taux de consommation des ménages est particulièrement élevé. Mais se pose alors la question de savoir comment réagiraient les employeurs à cette hausse des salaires qui réduirait nécessairement leurs bénéfices. II se pourrait, bien sûr, que les entreprises s'en accommodent tout simplement. Néanmoins, seules seraient en mesure d'accepter une telle compression les entreprises les mieux positionnées sur leur marché ; or en règle générale, celles-ci pratiquent déjà des salaires relativement élevés. La plupart des entreprises concernées par l'instauration de salaires minima n'auraient donc d'autre issue que de procéder à une hausse de leurs prix. Or en Allemagne, les bas salaires se concentrent sur quelques secteurs, principalement dans le tertiaire : dans la sécurité, et plus généralement dans les services de proximité comme l'hôtellerie/gastronomie, la teinturerie, les taxis ou les coiffeurs. L'instauration de salaires minima concernerait alors essentiellement de petites entreprises - et ce, à l'est bien plus qu'à l'ouest (graphique 2).

Or si ces entreprises sont contraintes d'augmenter leurs prix, comment réagira la demande ? II est très improbable que cette hausse laissera leurs clients indifférents. Et il faut bien plutôt redouter que si, en termes nominaux, la demande en demeurera inchangée, c'est par contre le volume des biens achetés qui devrait diminuer. Les clients continueront peut-être d'aller aussi souvent chez leur coiffeur malgré la hausse des prix, mais pour compenser leurs dépenses accrues, ils prendront moins souvent le taxi ou iront moins au cinéma. De ce fait, l'activité se réduira dans certains secteurs puisque, du fait du recul de la demande, les entre-
Les conclusions varient en effet selon la modélisation utilisée

Effets positifs des salaires minima sur la demande intérieure?

Les entreprises du tertiaire seraient contraintes à hausser leurs prix

Quel impact sur la demande? 
prises auront besoin de moins de main-d'œuvre. Ils se pourrait aussi que ces dernières répondent à la hausse des salaires par un effort accru de rationalisation qui, lui aussi, tasserait l'activité.

Graphique 2 : Part des salariés *) dans le secteur des bas salaires par rapport au total des salariés et par taille des entreprises en 2008

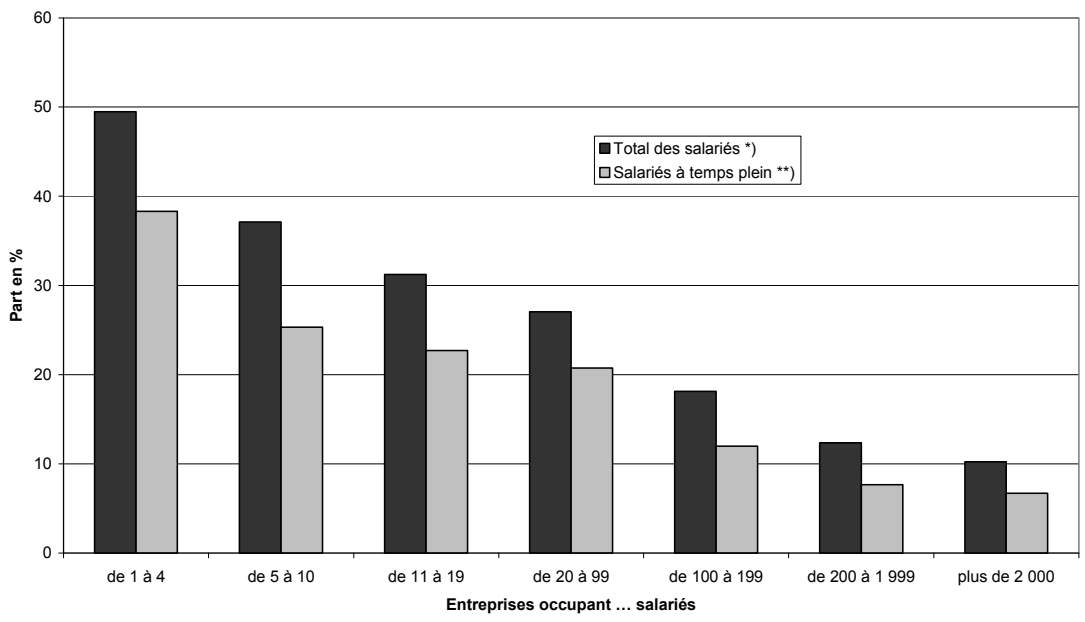

Source: SOEP, calculs de l'auteur. *) hors apprentis, chômeurs occupant un « job à $1 €$ », jeunes appelés ou jeunes effectuant leur service civil ; ${ }^{*}$ ) hors retraités, chômeurs enregistrés, lycéens et étudiants occupés.

Différencier par types de biens ou produits consommés

Quel impact sur l'échelle des salaires?
D'un autre côté, certains salariés verraient leur revenu disponible augmenter sous l'effet de l'instauration de salaires minima, et ils consommeront plus. Or dans ce cas, la question est de savoir quels sont les biens auxquels bénéficiera cette hausse de la demande. S'il s'agit de services primaires intensifs en maind'œuvre, la baisse de la consommation induite par la hausse des prix pourrait être en quelque sorte compensée. S'il s'agit par contre de produits de l'électronique grand public fabriqués en Asie, une demande accrue n'aurait que peu d'impact sur le marché domestique.

Enfin, il est probable que l'instauration de salaires minima en Allemagne n'entraînera pas seulement une hausse des salaires pour ceux qui étaient rémunérés à un niveau inférieur, mais qu'elle aura également des répercussions sur une large partie de la structure même des salaires. Si par exemple en Allemagne de l'est, une vendeuse auxiliaire perçoit un salaire minimum horaire de $7,50 €$ au lieu des $7,00 €$ en vigueur, la vendeuse qualifiée qui, elle, gagne actuellement $8,00 €$, serait en droit d'exiger une hausse afin de rétablir l'échelle précédente. Voilà quelques-unes des innombrables questions qu'il conviendrait de prendre en considération lorsqu'on cherche à évaluer à sa juste mesure l'impact des salaires minima sur l'emploi. Or cela est scientifiquement impossible.

AUCUN DES ARGUMENTS AVANCÉS DANS LE DÉBAT ALLEMAND sur les salaires minima n'est réellement convaincant. L'approche sous l'angle de la politique sociale est totalement déconnectée du réel. En effet, il n'y a que très peu de salariés qui, bien qu'ils travaillent à temps plein, sont tributaires d'un soutien versé par l'Etat sous forme de transferts sociaux parce qu'ils sont les seuls à faire vivre leur famille. Les bas salaires concernent avant tout les salariés occupant un emploi atypique, ainsi que les personnes à la recherche d'un revenu complémentaire, qu'ils soient retraités, étudiants ou chômeurs.

Tout aussi peu probant est l'argument selon lequel il faudrait instaurer des salaires minima afin de contenir le développement de l'inégalité salariale. Or le meilleur moyen pour réduire les écarts réside dans la croissance de l'emploi et la réduction du chômage. Car ainsi, les salariés verront leur position renforcée pour mener les négociations salariales. Certes, les chances d'accès à l'emploi sont inégalement réparties ; en Allemagne comme dans tous les autres pays industrialisés, ce sont ceux n'ont pas de qualification qui rencontrent les plus grandes diffi- 
cultés à l'insertion. Et ce n'est assurément pas l'instauration de salaires minima qui les résoudra. La France, dans le cadre de la réduction générale du temps de travail, a mené une politique de subvention des salaires, entre autres pour adapter les SMIC aux 35 heures sans pénaliser les employeurs. On peut être tenté d'en conclure que cela a généré une hausse de l'emploi - mais c'est ne pas tenir compte du coût élevé pour les budgets publics. Assurément, affectés autrement, les mêmes moyens auraient créé eux aussi des emplois. Mais ce qui est problématique avant tout, c'est qu'une politique de subventions salariales ne traite pas le mal à la racine. Comme c'est le manque de qualifications qui limite les chances d'accès à l'emploi, la meilleure solution serait plutôt d'investir plus dans le capital humain, donc dans la formation des jeunes générations.

Plusieurs années durant, on a débattu en Allemagne aussi de l'adoption d'une politique de subvention salariale (Kombilohn), avant de rejeter cette idée, considérant que ses effets sur l'emploi étaient sans commune mesure avec l'ampleur des moyens budgétaires qu'elle aurait requis. Les seules mesures qu'on ait conservées sont les mini-jobs - mais avant tout pour des raisons politiques, car elles s'inscrivent en rupture avec la doctrine en matière de prélèvements fiscaux et sociaux, puisque de facto l'Etat accorde à ces petits boulots un régime favorisé. De même, en matière de politique économique, ils se traduisent non seulement par une distorsion de la concurrence par rapport à l'emploi régulier ; ils contribuent aussi à tirer vers le bas les salaires dans l'emploi régulier, soumis à cotisations sociales. II conviendrait dès lors de mettre fin à cette mesure.

N'est pas défendable non plus l'argument selon lequel les salaires minima détruiraient des emplois - en tout cas, la science n'est pas en mesure de le valider, ni par la théorie ni par l'empirie. Cela étant, le risque est plausible. Car incontestablement, rémunération et productivité sont étroitement liées. II convient de toujours garder ce lien présent à l'esprit. Surtout en ce qui concerne l'Allemagne, un pays qui présente de fortes disparités régionales en matière de productivité, particulièrement entre les anciens et nouveaux Länder. Malgré les progrès réalisés dans l'ex-RDA, la productivité est-allemande est loin d'avoir atteint le niveau de l'ouest. Et si on instaurait en Allemagne des salaires minima généralisés, uniformes, il faudrait augmenter les salaires d'un grand nombre d'actifs. Or une hausse des bas salaires modifierait profondément, par effet domino, la structure de tous les salaires, les salariés qualifiés cherchant à rétablir l'écart salarial avec les moins qualifiés. Les conséquences sur les prix, la demande et l'emploi sont certes délicates à calculer, mais on peut légitimement estimer qu'elles seraient énormes. Instaurer par la loi des salaires minima uniformes et généralisés en Allemagne relèverait d'une expérience sur le terrain hautement risquée.

Quoi qu'il en soit, l'introduction de salaires minima légaux serait un péché capital en termes de doctrine économique et sociale. Celle-ci repose, depuis la constitution de la République fédérale, sur le principe unanimement reconnu que l'Etat n'a pas à intervenir dans l'autonomie tarifaire des partenaires sociaux - elle est garantie par la Loi fondamentale -, et donc dans le processus de fixation des salaires. Or ce consensus a été rompu unilatéralement par quelques syndicats isolés et les partis de gauche. Mais si l'Etat détermine à l'avenir un salaire plancher, les syndicats n'auront plus à assurer la défense des actifs dans le segment des bas salaires. Ils courent dès lors le risque de se transformer en organisations défendant les seuls intérêts catégoriels de certaines professions. Dans le segment des bas salaires, ce n'est plus le patronat qui sera l'interlocuteur des syndicats, mais l'Etat. Et en cas de besoin, c'est à l'Etat - donc à la collectivité des contribuables - qu'ils feront porter la responsabilité d'apporter son soutien sous forme de subventions salariales. Soit dit en passant, les salaires minima sont un péché capital contre la doctrine ordolibérale sous un autre angle aussi : l'expérience allemande a montré qu'ils peuvent servir d'instrument pour se protéger d'une concurrence indésirable - avec la bénédiction alors conjointe des mouvements patronal et syndical.

Traduction : I. Bourgeois 


\section{Indications bibliographiques}

- BACHmann Ronald, BAUER Thomas K., KLUVE Jochen, Mindestlöhne in Deutschland. Beschäftigungswirkungen und fiskalische Effekte, RWI-Materialien, $n^{\circ}$ 43, 2008

- BRENKE Karl, «Reallöhne in Deutschland über mehrere Jahre rückläufig », DIW-Wochenbericht, $n^{\circ} 33 / 2009$

- BRENKE Karl, « Jahrelanger Trend zunehmender Lohnspreizung gestoppt », DIW-Wochenbericht, $n^{\circ} 38 / 2008$

- BUNDESAgentur für ARBEIT, Aktuelle Daten aus der Grundsicherung. Erwerbstätigkeit von erwerbsfähigen Leistungsbeziehern, juin 2009

- KöNIG Marion, MöLLER Joachim, Mindestlohneffekte des Entsendegesetzes ? Eine Mikrodatenanalyse für die deutsche Bauwirtschaft, IAB Discussion Paper, № 30/2007

- MeYER Jörn-A. et al, Auswirkungen eines Mindestlohns auf kleine und mittlere Unternehmen. Eine betriebswirtschaftliche Analyse nach Branchen, Betriebstypen und Standorten. Studie im Auftrag der Friedrich-Ebert-Stiftung, 2007

- MüLLER Kai-Uwe, STEINER Viktor, « Mindestlöhne kosten Arbeitsplätze : Jobverluste vor allem bei Geringverdienern ", DIW-Wochenbericht, $n^{\circ}$ 30/2008

- NeUmark David, WAscher William, Minimum Wages and Employment, IZA Discussion Paper, $N^{\circ} 2570$ (2007)

- RAGNITZ Joachim, THum Marcel, «Beschäftigungswirkungen von Mindestlöhnen - eine Erläuterung zu den Berechnungen des ifo Instituts ", ifo-Schnelldienst, $n^{\circ}$ 1/2008

- STURM Simon, "Beschäftigungseffekte des französischen Mindestlohns », in HoRN Gustav A., JoEbges Heike, Logeay Camille, StURm Simon, Frankreich : Ein Vorbild für Deutschland? Ein Vergleich wirtschaftspolitischer Strategien mit und ohne Mindestlohn, IMK-Report, $\mathrm{n}^{\circ} 31$ (2008) 